\title{
Cobalt Metal-Organic Framework Based on Layered Double Nanosheets for Enhanced Electrocatalytic Water Oxidation in Neutral Media
}

\author{
Silvia Gutiérrez-Tarriño, José Luis Olloqui-Sariego, Juan José Calvente, Guillermo Mínguez Espallargas, \\ Fernando Rey, Avelino Corma, and Pascual Oña-Burgos*
}

Cite This: J. Am. Chem. Soc. 2020, 142, 19198-19208

Read Online

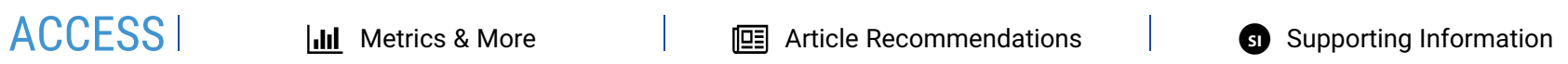

ABSTRACT: A new cobalt metal-organic framework (2D-Co-MOF) based on well-defined layered double cores that are strongly connected by intermolecular bonds has been developed. Its 3D structure is held together by $\pi-\pi$ stacking interactions between the labile pyridine ligands of the nanosheets. In aqueous solution, the axial pyridine ligands are exchanged by water molecules, producing a delamination of the material, where the individual double nanosheets preserve their structure. The original 3D layered structure can be restored by a solvothermal process with pyridine, so that the material shows a "memory effect" during the delamination-pillarization process. Electrochemical activation of a 2DCo-MOF@Nafion-modified graphite electrode in aqueous solution improves the ionic migration and electron transfer across the film and promotes the formation of the electrocatalytically active cobalt species for

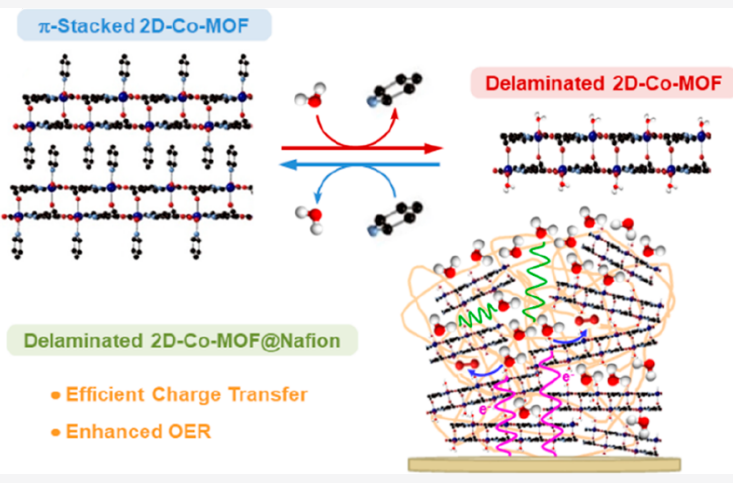
the oxygen evolution reaction (OER). The so-activated 2D-Co-MOF@Nafion composite exhibits an outstanding electrocatalytic performance for the OER at neutral $\mathrm{pH}$, with a TOF value $\left(0.034 \mathrm{~s}^{-1}\right.$ at an overpotential of $\left.400 \mathrm{mV}\right)$ and robustness superior to those reported for similar electrocatalysts under similar conditions. The particular topology of the delaminated nanosheets, with quite distant cobalt centers, precludes the direct coupling between the electrocatalytically active centers of the same sheet. On the other hand, the increase in ionic migration across the film during the electrochemical activation stage rules out the intersheet coupling between active cobalt centers, as this scenario would impair electrolyte permeation. Altogether, the most plausible mechanism for the $\mathrm{O}-\mathrm{O}$ bond formation is the water nucleophilic attack to single $\mathrm{Co}(\mathrm{IV})$-oxo or Co(III)-oxyl centers. Its high electrochemical efficiency suggests that the presence of nitrogen-containing aromatic equatorial ligands facilitates the water nucleophilic attack, as in the case of the highly efficient cobalt porphyrins.

\section{INTRODUCTION}

Water splitting is one of the most important processes for many applications associated with carbon-free energy storage and conversion. ${ }^{1-3}$ Water oxidation (WO) or the oxygen evolution reaction (OER) is a more complex transformation than proton reduction, and for this reason it is still considered the most challenging step in water splitting. ${ }^{4,5}$ In fact, the OER is crucial to produce oxygen from water successfully, 6,7 as well as for other applications such as regenerating fuel cells ${ }^{8}$ and rechargeable metal-air batteries. ${ }^{9}$

Precious-metal oxides $\mathrm{IrO}_{2}$ and $\mathrm{RuO}_{2}$ are, so far, the most efficient OER electrocatalysts, but their high cost, scarcity, and low durability make them impractical for large-scale applications. ${ }^{10,11}$ Other OER catalysts (mostly metal oxides/hydroxides) ${ }^{12}$ generally show unsatisfactory catalytic activities with large overpotentials..$^{10,11,13-15}$ Cobalt-based water oxidation electrocatalysts (WOC), including molecular complexes ${ }^{5,16,17}$ and inorganic nanoparticles, ${ }^{18-22}$ are of great interest due to their notable OER activities and abundance of this metal in the earth. ${ }^{3,23-25}$ MOFs are a novel class of porous materials that are emerging in the research of electrochemical water splitting due to their large surface areas, controllable arrangement of isolated active sites, and high design flexibility. ${ }^{26,27}$ However, few works have reported on the construction of cobalt-based MOFs for the electrocatalytic OER, ${ }^{28-41}$ despite their large structural features. These types of materials have typically suffered from low conductivity and low electrocatalytic activity; therefore, a large number of research efforts have been made to overcome these limitations. Strategies reported to facilitate the charge transport across the MOF include an increase in the charge delocalization

Received: August 18, 2020

Published: October 30, 2020 

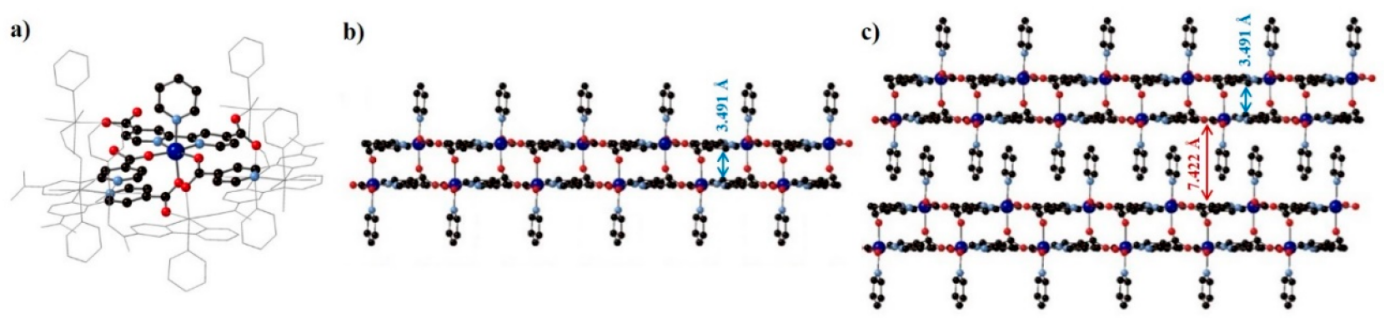

Figure 1. X-ray structure of (a) the secondary building unit, (b) a layered double nanosheet, and (c) $\pi-\pi$ stacked two-layered double nanosheets of 2D-Co-MOF. The blue arrow indicates the distance between the two single layers of a nanosheet $(3.491 \AA)$, and the red arrow indicates the intersheet distance between $\pi-\pi$ stacked nanosheets $(7.422 \AA)$.

by introducing donor-acceptor type interactions, ${ }^{42-45}$ mixed valent states of the node/linker, ${ }^{46,47}$ and $\pi-\pi$ stacking or $\pi$ conjugation into the framework. ${ }^{48}$ In addition, effective strategies to enhance the intrinsic electrocatalytic activity of MOFs have been focused on an increase in the accessibility of the active sites ${ }^{36-38}$ and modulation of their electronic environment. $^{35,39-41,49}$ The optimization of coordinative unsaturated metal sites of MOFs has been achieved by tuning the synthetic protocol ${ }^{38,49,50}$ and/or post-treatment methods via MOF activation by solvent-assisted ligand exchange, ${ }^{49}$ plasma engraving, $^{36,51}$ or electrochemical activation. ${ }^{32,38-40}$ Furthermore, electronic structures of the building units of MOFs have been modulated by incorporating missing linker and missing node defects ${ }^{37,49,52-54}$ or by the construction of hybrid MOF heterostructures containing two or more different kinds of metal ions or organic linkers. ${ }^{34,35,39-41}$ Because of the intrinsically high kinetic barrier for the OER, most of the reported cobalt-based MOF electrocatalysts operate in strongly alkaline media, which implies very highly corrosive and harsh conditions for large-scale applications. Hence, a major challenge is the development of new MOFs for efficient electrocatalytic water oxidation at neutral $\mathrm{pH}$.

In addition, previous studies have shown that the electrocatalytically active species for the cobalt-mediated OER are the $\mathrm{Co}(\mathrm{IV})$-oxo or $\mathrm{Co}$ (III)-oxyl radical species generated from the resting state of the cobalt centers by proton-coupled electron transfers. In this sense, different mechanisms have been proposed for the cobalt-mediated $\mathrm{O}-\mathrm{O}$ bond formation, namely: (i) direct coupling between two oxo or oxyl ligands located in distinct cobalt centers, which produces a bridging peroxo intermediate, ${ }^{5,56}$ (ii) geminal coupling of an oxo (or oxyl) ligand with another water-derived ligand coordinating the same cobalt center, ${ }^{57,58}$ and (iii) water nucleophilic attack to the oxo or oxyl radical ligands, generating the corresponding hydroperoxide intermediate. ${ }^{59-61}$ Unfortunately, despite previous efforts, the exact mechanism is still unresolved.

Herein, a new cobalt MOF based on well-defined layered double cores (2D-Co-MOF) has been synthesized. These layered double cores are strongly connected by intermolecular bonds, holding its $3 \mathrm{D}$ structure together by $\pi-\pi$ stacking interactions between the labile pyridine ligands of the nanosheets. Treatment of this $\pi$-stacked 2D-Co-MOF with water provokes the exchange of the axial labile pyridine ligands by water molecules that triggers the delamination of the material, producing double nanosheets (delaminated 2D-CoMOF), which opens up the formation of active cobalt sites for their application in the OER. In addition, its dispersion in Nafion provides a composite (2D-Co-MOF@Nafion) with a good adherence to graphite electrodes suitable for the electrocatalytic water oxidation reaction. Moreover, electrochemical activation of the composite facilitates both ionic migration and electron transfer across the film, which in turn generates more open active cobalt sites for water oxidation. The fully activated 2D-CoMOF@Nafion composite exhibits superior electrocatalytic performance for the OER at neutral $\mathrm{pH}$ in comparison to similar nanosheet-based materials reported in the literature. This enhanced electrocatalytic activity can be ascribed to the presence of nitrogen-containing aromatic equatorial ligands that promote the water nucleophilic attack as in the case of the highly efficient cobalt porphyrins and related systems commonly employed as catalysts for the homogeneous water oxidation reaction. $^{62,63}$

\section{RESULTS AND DISCUSSION}

Synthesis and Characterization of the $\pi-\pi$ Stacked 2D-Co-MOF. The new 2D-Co-MOF was obtained by solvothermal synthesis at $150{ }^{\circ} \mathrm{C}$ for 9 days. The cubane cobalt cluster $\left[\mathrm{Co}_{4} \mathrm{O}_{4}(\mathrm{OAc})_{4}(\mathrm{py})_{4}\right]^{64}$ has been seen to be a key for the synthesis of the new 2D-Co-MOF material, since attempts to obtain this new material with other common reagents such as $\mathrm{Co}\left(\mathrm{NO}_{3}\right)_{2} \cdot 6 \mathrm{H}_{2} \mathrm{O}$ and $\mathrm{Co}(\mathrm{OAc})_{2}$ were unsuccessful under a wide range of different synthetic conditions (Figure $\mathrm{S} 1$ and Table S1). The solvothermal reaction of $\left[\mathrm{Co}_{4} \mathrm{O}_{4}(\mathrm{OAc})_{4}(\mathrm{py})_{4}\right]$ and 2,2'-bipyridine-4,4'-dicarboxylic acid $\left(\mathrm{H}_{2} \mathrm{bda}\right)$ in pyridine results in the formation of red crystals of 2D-Co-MOF. Singlecrystal X-ray diffraction reveals that 2D-Co-MOF crystallizes in the monoclinic $P 2_{1} / n$ space group (Figure S2 and Table S2). The $\mathrm{Co}^{2+}$ atom lies in a distorted-octahedral environment (Figure 1a and Figure S3) and is coordinated by three oxygen atoms from three different $\mathrm{bda}^{2-}$ ligands, one of them in an axial position ( $\mathrm{sp}^{2}$ oxygen of the carboxylic group) and the other two which take up two of the equatorial positions (alkoxy substituent of the carboxylic group). These carboxylate groups are chelating monodentate, and each oxygen of a carboxylic group is coordinated with a different cobalt center. Each $\mathrm{Co}^{2+}$ ion is also coordinated in a bidentate fashion to two nitrogen atoms of one $\mathrm{bda}^{2-}$ ligand, different from the three which are coordinated to the cobalt by the carboxylic group, and to a nitrogen atom from a pyridine ligand, which occupies the second axial position to form six-coordinate metal ions (Figures S4 and S5).

This material extends in two directions of the space, forming a 3D layer material consisting of nanosheets comprised of two single layers with an interplanar distance of $3.491 \AA$ (Figure $1 \mathrm{~b}$ and Figure S6). Consecutive nanosheets are further packed into three-dimensional supramolecular structures through interlayer face to face $\pi \cdots \pi$ interactions between pyridine ligands (Figure $1 \mathrm{c}$ and Figure S7). The distance between the centroids of the pyridine rings is $4.238 \AA$. Two cobalt atoms of two different layers are connected by two carboxylate groups of two different 
a

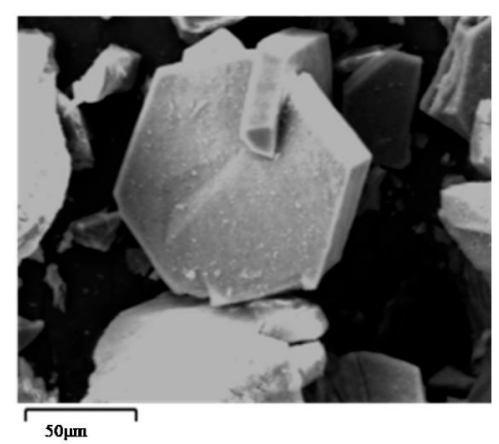

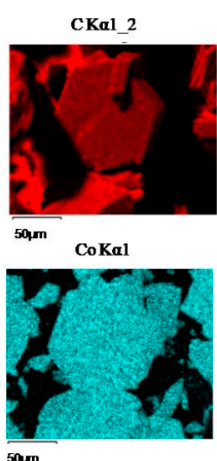

soum

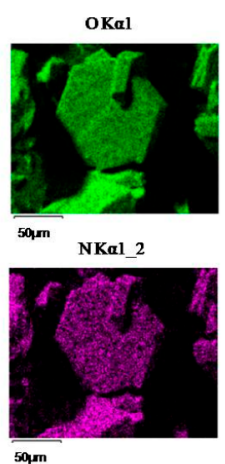

d

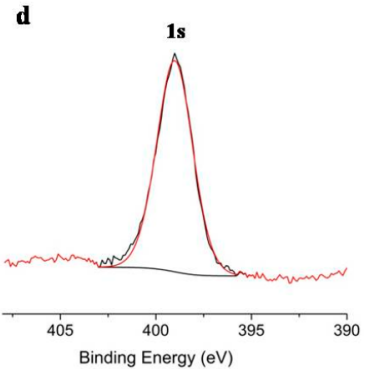

$50,4 m$ b

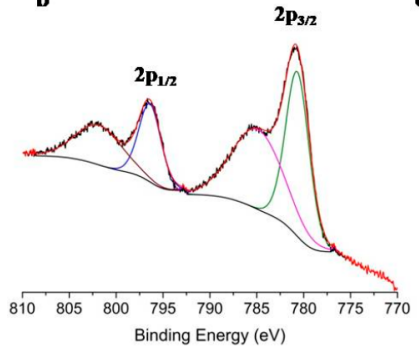

c

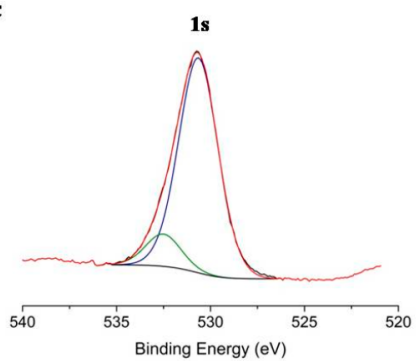

Figure 2. (a) FESEM image of 2D-Co-MOF and its EDX analysis. XPS spectra of (b) Co 2p line, (c) O 1s line, and (d) N 1s line of 2D-Co-MOF.
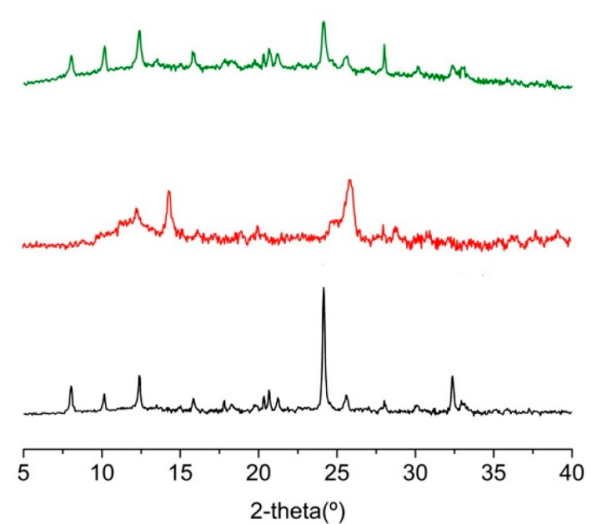

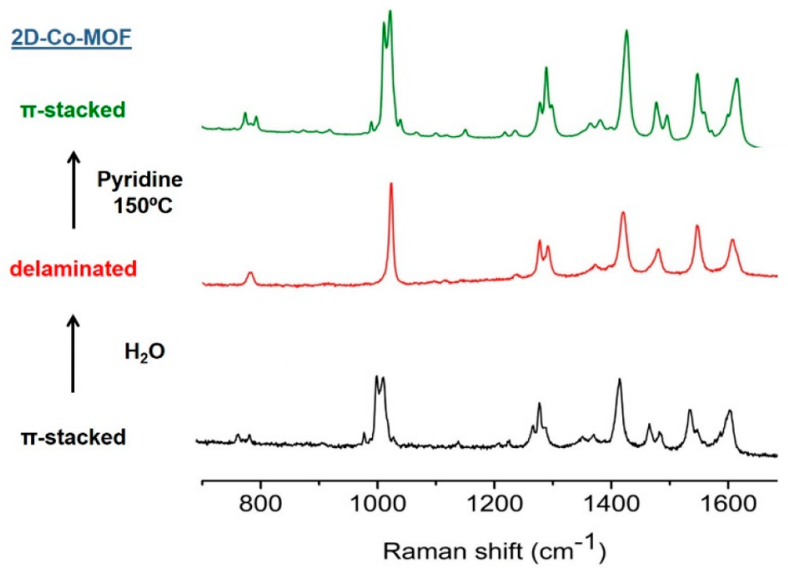

Figure 3. Powder X-ray diffraction patterns (left panel) and Raman spectra (right panel) of the $\pi$-stacked 2D-Co-MOF before (black) and after successive treatment with $\mathrm{H}_{2} \mathrm{O}$ for $5 \mathrm{~min}$ (red) and with pyridine for 2 days at $150{ }^{\circ} \mathrm{C}$ (green).

$\mathrm{bda}^{2-}$ ligands, the distance between these two cobalt ions being $4.69 \AA$ A.

The chemical analysis of this new material was determined by elemental analysis (EA) and inductively coupled plasma atomic emission spectroscopy (ICP-AES). The obtained results (C, 55.07; H, 3.05; N, 10.77; Co, 15.05) match well with the formula $\mathrm{C}_{18} \mathrm{H}_{11} \mathrm{CoN}_{3} \mathrm{O}_{4}$ determined by single-crystal X-ray diffraction (more information is given in the Supporting Information). The thermal stability was tested by thermogravimetric analysis (Figure S8), which is characterized by three mass loss steps. The first one at $120{ }^{\circ} \mathrm{C}$ is ascribed to the solvent enclosed in the material, whereas the other two overlapped steps at around 400 ${ }^{\circ} \mathrm{C}$ correspond to the loss of pyridine and bda ligands (more details are given in the Supporting Information). The microporosity of the material has been demonstrated upon activation at $100{ }^{\circ} \mathrm{C}$ under vacuum, with a moderate $\mathrm{CO}_{2}$ adsorption capacity of $1.1 \mathrm{mmol} / \mathrm{g}$ at $0{ }^{\circ} \mathrm{C}$ and $100 \mathrm{kPa}$ (Figure S9a,b), which corresponds to an apparent surface area of $61 \mathrm{~m}^{2} / \mathrm{g}$.
In order to provide further composition characterization, 2DCo-MOF was studied by FESEM (field emission scanning electron microscopy) and EDX (energy dispersive X-ray spectroscopy) analysis (Figure 2a). 2D-Co-MOF crystals have a hexagonal morphology, and all of its elements are well distributed, as can be observed in the EDX images.

The electronic structure of this material was also analyzed by $\mathrm{X}$-ray photoelectron spectroscopy (XPS) (Figure $2 \mathrm{~b}-\mathrm{d}$ ) to elucidate the nature of the coordination environment. The survey spectrum shows the presence of cobalt, oxygen, carbon, and nitrogen. The analysis of XPS spectra of $2 p$ transition metals is not a straightforward process, as they contain multiplet splitting and shakeup structures that may prove difficult in the identification of the chemical states present. ${ }^{65}$ The binding energy $\left(E_{\mathrm{b}}\right)$ value is typically used in XPS to determine the oxidation number of a chemical element, though for the $3 \mathrm{~d}$ transition elements the spin-orbit splitting is more informative. According to theoretical calculations, ${ }^{66}$ the Co $2 \mathrm{p}_{1 / 2}-$ Co $2 \mathrm{p}_{3 / 2}$ spin-orbit splitting increases when the number of the unpaired 

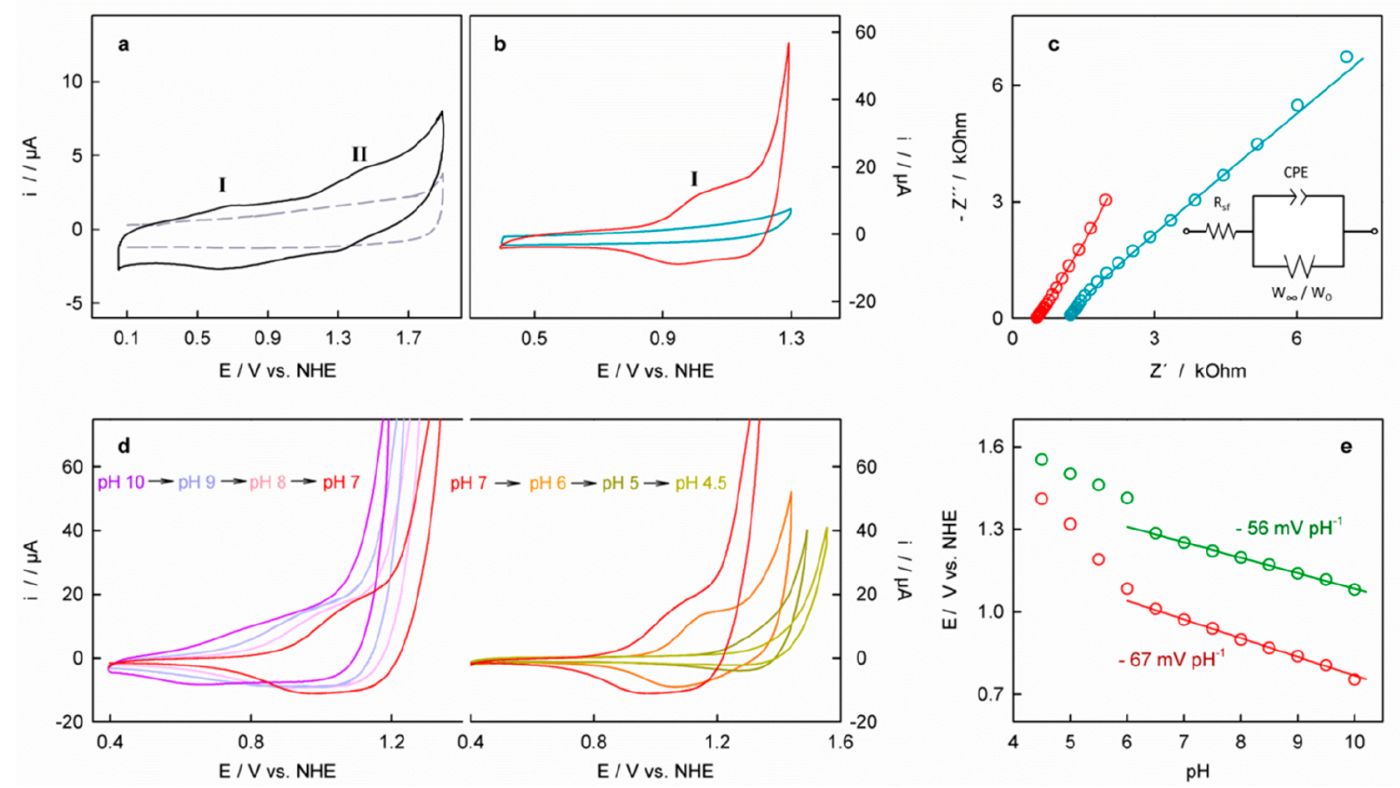

Figure 4. (a) Cyclic voltammogram of a pyrolytic graphite electrode modified with Nafion (gray dashed line) or with 2D-Co-MOF@Nafion (black line) recorded at $0.05 \mathrm{~V} \mathrm{~s}^{-1}$ in acetonitrile. (b) Cyclic voltammograms recorded at $0.05 \mathrm{~V} \mathrm{~s}^{-1}$ in aqueous $0.1 \mathrm{M} \mathrm{SPB}$ solution pH 7 at $25^{\circ} \mathrm{C}$ before $($ blue line) and after (red line) electrochemical activation of 2D-Co-MOF@Nafion deposited on a pyrolytic graphite electrode and (c) corresponding Nyquist plots measured at $1.1 \mathrm{~V}$ vs NHE, before (blue symbols) and after (red symbols) electrochemical activation. Solid lines are the best fits using the equivalent circuit of the inset plot with the parameter values reported in Table S5. (d) Cyclic voltammograms of a pyrolytic graphite electrode modified with 2D-Co-MOF@Nafion recorded at $0.05 \mathrm{~V} \mathrm{~s}^{-1}$ and $25^{\circ} \mathrm{C}$ in an aqueous $0.1 \mathrm{M}$ SPB solution at the indicated $\mathrm{pHs}$ and (e) the corresponding $E$ vs $\mathrm{pH}$ plot estimated at $8 \mu \mathrm{A}$ (red symbols) and $40 \mu \mathrm{A}$ (green symbols).

$3 \mathrm{~d}$ electrons is increased, being closer to $16 \mathrm{eV}$ for high-spin $\mathrm{Co}(\mathrm{II})$ and to $15 \mathrm{eV}$ for $\mathrm{Co}(\mathrm{III})$. For the current 2D-Co-MOF, $E_{\mathrm{b}}\left(\right.$ Co $\left.2 \mathrm{p}_{3 / 2}\right), E_{\mathrm{b}}\left(\right.$ Co $\left.2 \mathrm{p}_{1 / 2}\right)$, and $\Delta E_{1}$ values are $780.7,796.4$, and $15.7 \mathrm{eV}$, respectively (Figure $2 \mathrm{~b}$ ). Intense satellite peaks $\left(\sum \mathrm{sat} / \mathrm{ICo}_{2 \mathrm{p}}>1.6\right)$ have been observed for the Co $2 \mathrm{p}_{3 / 2}$ spectra. Both $\sum \mathrm{sat} / \mathrm{ICo}_{2 \mathrm{p}}$ and $\Delta E_{1}$ values are characteristic of the highspin cobalt(II) compounds, ${ }^{67-70}$ indicating that the cobalt centers of 2D-Co-MOF are exclusively in the Co(II) oxidation state. In addition, the $\mathrm{O} 1 \mathrm{~s}$ peak was deconvoluted into two components at 531.1 and $532.6 \mathrm{eV}$ corresponding to $\mathrm{O}=\mathrm{C}-\mathrm{O}$ and $\mathrm{C}-\mathrm{O}$, respectively (Figure $2 \mathrm{c}$ ). Finally, the $\mathrm{N} 1 \mathrm{~s}$ peak presents only one contribution at $399 \mathrm{eV}$ characteristic of pyridinic nitrogen (Figure 2d).

Finally, the chemical stability of the new 2D-Co-MOF material in different solvents was investigated from the powder diffraction patterns measured after contacting this material with protic solvents such as ethanol and water, nonprotic solvents such as acetonitrile, and strongly alkaline solvent such as triethylamine ( $\mathrm{pH}$ 12.7) (Figures S10 and S11). The X-ray powder diffraction spectra reveal that this material maintains its structure intact after being treated with ethanol, acetonitrile, and triethylamine at $25{ }^{\circ} \mathrm{C}$ over 1 day. However, treatment with water produces significant structural changes, as described in the next section.

Reversible Chemical Delamination of the $\pi-\pi$ Stacked 2D-Co-MOF. In order to assess the chemical stability of the sodeveloped cobalt MOF in water for its application to promote the oxygen evolution reaction, further experiments have been carried out to probe their structural implications. In particular, Raman, PXRD, and XPS spectra of the $\pi$-stacked 2D-Co-MOF have been measured before and after its treatment with water for $5 \mathrm{~min}$. As can be seen in the Raman spectra depicted in Figure 3, the most striking findings in the presence of water (red spectrum) is the loss of the Raman bands associated with the axially coordinated pyridine ligands $(768,1010$, and 1283 $\mathrm{cm}^{-1}$ ), and the insensitivity of the bands associated with the bipyridine $\left(774,1022\right.$, and $1278 \mathrm{~cm}^{-1}$ ) and the carboxylic groups $\left(1289,1426,1546\right.$, and $\left.1615 \mathrm{~cm}^{-1}\right)$, indicating that the cobalt centers preserve their coordination to the bda and carboxylic ligands after treatment with water. Interestingly, the XPS spectrum of the 2D-Co-MOF after treatment with water reveals the presence of a shoulder at $532.8 \mathrm{eV}$ in the main $\mathrm{O} 1 \mathrm{~s}$ peak, which is ascribed to cobalt-coordinated water molecules, ${ }^{71}$ thereby suggesting that the axial labile pyridine ligand is replaced by water molecules (Figure S12). Additionally, the overall change of the PXRD pattern, with the loss or broadening of some characteristic bands (red spectrum in Figure 3), is consistent with a delamination of the $\pi$-stacked 3D structure of the MOF to produce individual nanosheets, whose double-layer structure is preserved.

Interestingly, we have found that the 3D structure of the delaminated 2D-Co-MOF can be regenerated by a solvothermal process with pyridine at $150{ }^{\circ} \mathrm{C}$ for 2 days (green spectra in Figure 3). This fact has been corroborated using XPS, where the shoulder at $532.8 \mathrm{eV}$ in the main $\mathrm{O} 1 \mathrm{~s}$ peak, which is ascribed to cobalt-coordinated water molecules, disappears after the solvothermal treatment with pyridine (Figure S14), indicating the coordination of the pyridines in the axial position again and the regeneration of the $\pi$-stacked 3D structure. Finally, the characterization of the regenerated $\pi$-stacked 2D-Co-MOF has been completed with elemental analysis and ICP, which confirm the same bulk composition of the regenerated material (Table S3).

To the best of our knowledge, this is the first time that MOFs have shown a "memory effect" in the delamination-pillarization process, which is well-known for the most famous $2 \mathrm{D}$ materials such as hydrotalcites. ${ }^{72}$ 
On the other hand, to assess the suitability of the sodeveloped 2D-Co-MOF as an electrocatalyst, we produced a composite by its dispersion in an alcoholic Nafion solution (2DCo-MOF@Nafion), which is a commonly used polymer in OER electrocatalysis. ${ }^{73}$ The influence of Nafion on the chemical stability of the $\pi$-stacked 3D structure of the MOF in water was also investigated by means of powder X-ray diffraction. From a comparison of the PXRD spectra of the 2D-Co-MOF measured before and after its treatment with water for different contact times (Figure S13), it was found that the $\pi$-stacked 2D-CoMOF adopts a preferential [100] orientation in the composite, where the crystals of the MOF interact in a planar way with the Nafion. In addition, in the presence of an aqueous solution, the $3 \mathrm{D}$ structure of the MOF only lasted about $40 \mathrm{~min}$ before suffering the delamination process. Thus, Nafion slows down the pyridine-water exchange but does not preclude it.

In addition, we have found that the current 2D-Co-MOF@ Nafion composite shows good adherence to graphite electrodes and long chemical stability for its application in promoting the electrocatalytic water oxidation reaction (vide infra).

Interplay between Redox Electrochemistry and Coordinative Chemistry of the 2D-Co-MOF@Nafion Composite. We first investigated the electrochemical redox conversion of the 2D-Co-MOF@Nafion composite in the absence of water by measuring the voltammetric response of a pyrolytic graphite electrode coated with the MOF composite in an acetonitrile solution containing $0.1 \mathrm{M}\left[\mathrm{Et}_{4} \mathrm{~N}\right] \mathrm{PF}_{6}$. As shown in Figure $4 \mathrm{a}$, its electrochemical response is characterized by two pairs of voltammetric waves located at $0.65 \mathrm{~V}$ (wave I) and $1.38 \mathrm{~V}$ (wave II) vs NHE, which correspond to the $\mathrm{Co}$ (II)/Co(III) and $\mathrm{Co}(\mathrm{III}) / \mathrm{Co}(\mathrm{IV})$ redox conversions, respectively. ${ }^{38}$

Electrochemical Activation in Aqueous Solutions. In an aqueous $0.1 \mathrm{M}$ sodium phosphate buffer solution (SPB) at $\mathrm{pH} 7$, observation of a significant cobalt redox conversion requires electrochemical activation of the so-modified electrode by applying a positive enough potential of $1.2 \mathrm{~V}$ (vs NHE) for at least $30 \mathrm{~min}$, as evidenced by comparing the voltammograms measured before (blue line) and after (red line) electrochemical activation (Figure $4 b$ ). As described above, the presence of the Nafion in the 2D-Co-MOF@Nafion composite slows down the pyridine-water exchange but does not preclude it. According to this finding, the cobalt centers of the delaminated 2D-CoMOF@Nafion-modified electrode equilibrated in the aqueous buffer solution are coordinated axially to water molecules. Thus, the initial electroinactivity can be tentatively ascribed to a limitation of the ionic migration across the film that is required to compensate for the interfacial charge developed during the redox conversion of the cobalt centers. This limitation may originate from the compactness of the hydrophobic domains of Nafion. To probe this possibility, we have measured the voltammetric features of distinct 2D-Co-MOF@Nafion composites differing in the Nafion content (Figure S21), observing that an increase of the Nafion content attenuates the incipient voltammetric features associated with the redox conversion of the cobalt centers and the OER. This finding corroborates the initial blocking properties of the Nafion film and suggests that the enhancement of the electrocatalytic current during the electrochemical activation of the composite might result from a decompaction of the film. On the other hand, for the electrochemically activated electrode, only the first cobalt voltammetric wave (at ca. $1.04 \mathrm{~V}$ vs $\mathrm{NHE}$ ) is well resolved due to concomitant contribution of the exponentially increasing electrocatalytic current associated with the oxygen evolution reaction. Bearing in mind that the electrocatalytically active species are the $\mathrm{Co}$ (IV)-oxo or $\mathrm{Co}$ (III)-oxyl radicals, ${ }^{56,63,74}$ the proximity of the OER current to the cobalt voltammetric wave indicates that the formation of the above species is favored in aqueous solution with respect to the formation of $\mathrm{Co}$ (IV) in acetonitrile. To get more information on the cobalt-mediated electrocatalytic oxidation of water, we measured the voltammetric response of the 2D-Co-MOF@Nafion composite for different acetonitrile/water ratios by adding variable volumes of an aqueous sodium phosphate buffer at $\mathrm{pH} 7$ to the initial acetonitrile solution in the electrochemical cell (Figure S22). The increase in the exponential-like voltammetric feature located at $E>1.3 \mathrm{~V}$ (vs NHE) with the water content clearly indicates that it corresponds to the electrocatalytic oxidation of water. On the other hand, the small hysteresis between the forward and backward currents reveals that the electrocatalytic process is partially limited by water diffusion, since otherwise either a sigmoidal (absence of mass transport control) or peaked (full mass transport control) voltammetric feature is expected. $^{75,76}$

Furthermore, analysis of the scan rate dependence of anodic wave I (Figure S23) reveals that the $\mathrm{Co}(\mathrm{II}) / \mathrm{Co}(\mathrm{III})$ redox conversion is fast for low scan rates $\left(\lesssim 0.5 \mathrm{~V} \mathrm{~s}^{-1}\right.$, surfaceconfined behavior) and becomes limited by the charge transport across the film for higher scan rates (diffusion-like behavior).

To get more insights into the effect of the electrochemical activation, electrochemical impedance spectra (EIS) of the immobilized 2D-Co-MOF@Nafion composite were measured at $1.1 \mathrm{~V}$ (vs NHE) before and after its electrochemical activation (Nyquist plots in Figure 4c). Before the electrochemical activation (blue symbols), the EI spectrum shows the typical shape expected for a diffusion-controlled redox process with a limiting linear segment of slope $\sim 1$ in the low-frequency region (high $Z^{\prime}$ values). This spectrum was quantitatively reproduced (solid line) with the equivalent circuit depicted in the inset of Figure $4 \mathrm{c}$ (or in Figure S25), where $R_{\mathrm{sf}}$ stands for the solution and film resistance, CPE for the constant phase element, and $W_{\infty}$ for the semi-infinite diffusion Warburg element (optimum fitting parameter values are reported in Table S5 and Figures S24 and S25 and the corresponding Bode plots in Figure S24). After electrochemical activation, the $x$ intercept of the spectrum (equal to $R_{\mathrm{sf}}$ ) shifted toward a lower $Z^{\prime}$ value, which reveals an increase in the ionic migration rate across the MOF, and the lowfrequency region showed an upward curvature, which is typical of a finite diffusion-controlled electron transfer across the film. ${ }^{77-81}$ This spectrum was quantitatively reproduced by the equivalent circuit depicted in the inset of Figure $4 c$ (or in Figure S25), provided that the semi-infinite diffusion $W_{\infty}$ element was replaced by the finite-diffusion element with a totally reflecting boundary $W_{\mathrm{o}}$ (optimum fitting parameter values are reported in Table S5). The change from semi-infinite to finite diffusion is consistent with an increase in the electron transfer rate across the film. Overall, these results reveal that electrochemical activation facilitates both ionic migration and electron transfer across the Co-MOF composite.

To explore whether the electrochemical activation leads to structural and/or coordinative changes, we have measured the Raman, PXRD, and XPS spectra, as well as the FESEM and FIB images and EDX analysis of the composite before and after its electrochemical activation at $1.2 \mathrm{~V}$ (vs NHE) for different exposition times, where an extensive OER takes place. The insensitivity of the Raman and PXRD spectra (Figures S16 and S17) to the electrolysis time reveals that the cobalt centers 
maintain their coordination sphere and the individual nanosheets preserve their double-layer structure during their electrochemical activation. XPS spectra (Figure S18 and Table S4) corroborate these findings and also reveal that electrochemical activation does not affect the oxidation state of the cobalt centers in their resting state $\mathrm{Co}(\mathrm{II})$, where the presence of $\mathrm{CoO}_{x}$ has not been detected. On the other hand, FESEM and FIB images (Figures S19 and S20) and the EDX analysis (Figure S19) clearly show that cobalt centers are homogeneously distributed in the composite, thus ruling out metal aggregation. These results reveal that delaminated double nanosheets retain their crystallinity and morphology during extensive OER.

Altogether, these findings suggest that the enhancement in the electrocatalytic activity during the electrochemical activation of the composite might originate from an increase in the ionic permeability of the film, as evidenced by a decrease in its electrical resistance determined by electrochemical impedance spectroscopy, rather than from induced structural changes. Along this line, we speculate that the generation of oxygen microbubbles during electrochemical water oxidation might contribute to the formation of microchannels in the film that facilitate the accessibility of the electrolyte to the cobalt centers.

Mechanistic Insights. The exchange of pyridine ligands by water molecules is expected to facilitate charge compensation in the electrochemical oxidation of the cobalt centers by ionization of the corresponding water-derived ligands. To probe this issue and provide a more complete redox speciation of the immobilized 2D-Co-MOF, we have studied the effect of the solution $\mathrm{pH}$ on its voltammetric response by using sodium phosphate buffer (Figure 4d). It should be noted that the 2DCo-MOF@Nafion-modified electrode was equilibrated in the aqueous buffer solution, so that the starting cobalt species was $\mathrm{Co}^{2+}\left(\mathrm{OH}_{2}\right)$. With an electrochemically activated 2D-CoMOF@Nafion in a 0.1 M SPB solution of pH 10 as the starting material, a decrease in the solution $\mathrm{pH}$ resulted in the shift of the cobalt voltammetric wave toward more positive potentials with a slope of ca. $-60 \mathrm{mV} / \mathrm{pH}$ up to $\mathrm{pH} 6.5$ (Figure $4 \mathrm{e}$ ). This linear dependence is typical of a redox conversion involving a protoncoupled electron transfer with the same number of exchanged protons and electrons. Bearing in mind that the $\mathrm{p} K_{\mathrm{a}}$ value of the water molecule coordinated to $\mathrm{Co}$ (III) in molecular complexes is within the range $5-7^{65,82,83}$ and that of water coordinated to $\mathrm{Co}(\mathrm{II})$ in polyoxometalates is greater than $10,{ }^{61,84}$ the above $\mathrm{pH}$ dependence is consistent with a deprotonation of the coordinating water molecule upon oxidation of the $\mathrm{Co}$ (II) centers according to

$$
\mathrm{Co}^{\mathrm{II}}-\mathrm{OH}_{2} \rightleftarrows \mathrm{Co}{ }^{\mathrm{III}}-\mathrm{OH}+\mathrm{e}^{-}+\mathrm{H}^{+}
$$

Below pH 6.0, a progressive, irreversible loss of the cobalt voltammetric wave occurs until it disappears at $\mathrm{pH}<5$. The concomitant decrease of OER current corroborates the catalytic role of the cobalt centers. Bearing in mind that the $\mathrm{p} K_{\mathrm{a}}$ values of the two carboxylic acid substituents of the bda ligand are 1.5 and $3.0^{85}$ and the $\mathrm{p} K_{\mathrm{a}}$ values of the two pyridyl nitrogens of $2,2^{\prime}$ bipyridine are 1.8 and $4.5,{ }^{86,87}$ the irreversible loss of the electrochemical activity can be ascribed to the MOF breakdown induced by protonation of the bda acid/base groups.

The voltammetric branch associated with the electrocatalytic oxidation of water shifts with the solution $\mathrm{pH}$ in a way similar to that for the cobalt voltammetric features (green symbols in Figure 4e), indicating that the cobalt-mediated electrocatalysis of the OER proceeds through a series of proton-coupled electron transfers with the same number of exchanged electrons and protons.

On the basis of the above electrochemical and spectroscopic results, the mechanism shown in Figure 5 for the redox

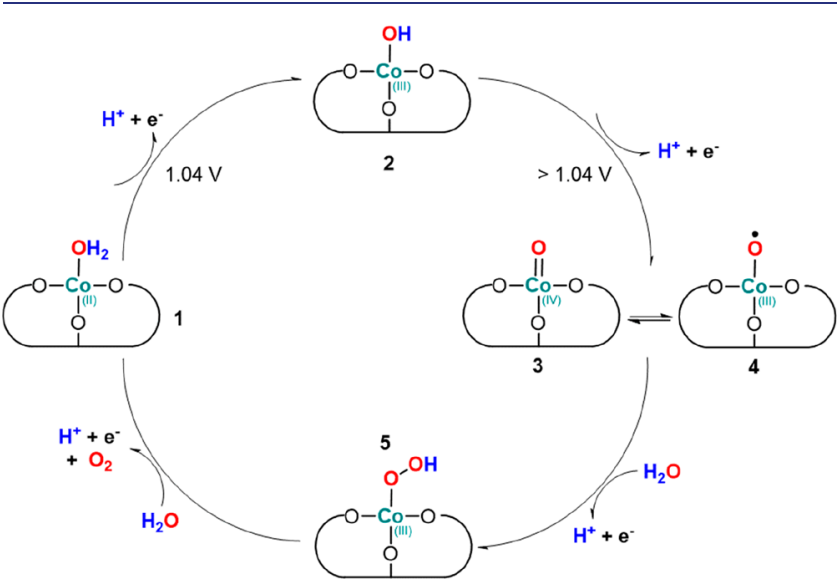

Figure 5. Proposed mechanism for the electrocatalytic oxygen evolution reaction mediated by $2 \mathrm{D}-\mathrm{Co}-\mathrm{MOF}$ at neutral $\mathrm{pH}$.

conversion and electrocatalytic water oxidation involving the 2D-Co-MOF@Nafion composite in aqueous solution is proposed. First, electrochemical oxidation of the cobalt centers $\left(\mathrm{Co}^{2+}-\mathrm{OH}_{2}, \mathbf{1}\right)$ at $\sim 1.04 \mathrm{~V}$ vs $\mathrm{NHE}$ is accompanied by ionization of the water ligand to produce $\mathrm{Co}^{3+}-\mathrm{OH}(2)$ via a proton-coupled electron transfer (PCET). Then, a second PCET at a somewhat more positive potential is expected to produce the oxo intermediate $\mathrm{Co}^{4+}=\mathrm{O}(3)$, which has a significant $\mathrm{Co}^{3+}$ oxyl radical character $\left(\mathrm{Co}^{3+}-\mathrm{O}^{\bullet}, 4\right)$, which has been recognized to be the catalytically active species for the OER. From the different mechanisms proposed for the cobaltmediated $\mathrm{O}-\mathrm{O}$ bond formation, ${ }^{55-61}$ direct coupling between two $\mathrm{Co}^{4+}=\mathrm{O}\left(\right.$ or $\mathrm{Co}^{3+}-\mathrm{O}^{\bullet}$ ) moieties of the same nanosheet is discarded because of the large Co...Co distance (4.69 $\AA$ ). The lack of a second water-derived ligand in the cobalt centers rules out the geminal coupling. On the other hand, the increase in the film ionic permeation during the electrochemical activation of the composite indicates that the OER electrocatalysis requires a good accessibility of the electrolyte to the cobalt centers that is fulfilled if delaminated nanosheets are well separated from each other. However, this scenario does not favor the intersheet radical coupling mechanism, as it requires quite small intersheet distances for the formation of the corresponding dinuclear moiety. This situation is in contrast to that found in cobalt oxide, where the radical coupling mechanism is a realistic scenario, as it involves two surface oxo bridged cobalt centers with good accessibility for the electrolyte. Since for the current system the above two requirements (proximity and electrolyte accessibility) cannot be simultaneously fulfilled, the more plausible scenario for the OER mechanism is the mononuclear water nucleophilic attack to $\mathrm{Co}^{4+}=\mathrm{O}$ or $\mathrm{Co}^{3+}-\mathrm{O}^{\bullet}$ to produce the hydroperoxide intermediate $\mathrm{Co}^{3+}-\mathrm{O}-\mathrm{OH}(5)$ via a $\mathrm{PCET}$, which may be facilitated by the bipyridine equatorial ligands as in the case of the highly efficient cobalt porphyrins. ${ }^{62}$ Finally, intermediate $\mathbf{5}$ reacts with water to form the oxygen molecule and regenerate the resting state of cobalt $\mathrm{Co}^{2+}-\mathrm{OH}_{2}$ through another PCET.

Benchmarking the Electrocatalytic Performance of the 2D-Co-MOF@Nafion Composite for the Water Oxidation Reaction. In order to assess the electrocatalytic activity of 2D-Co-MOF@Nafion composite toward the OER in 

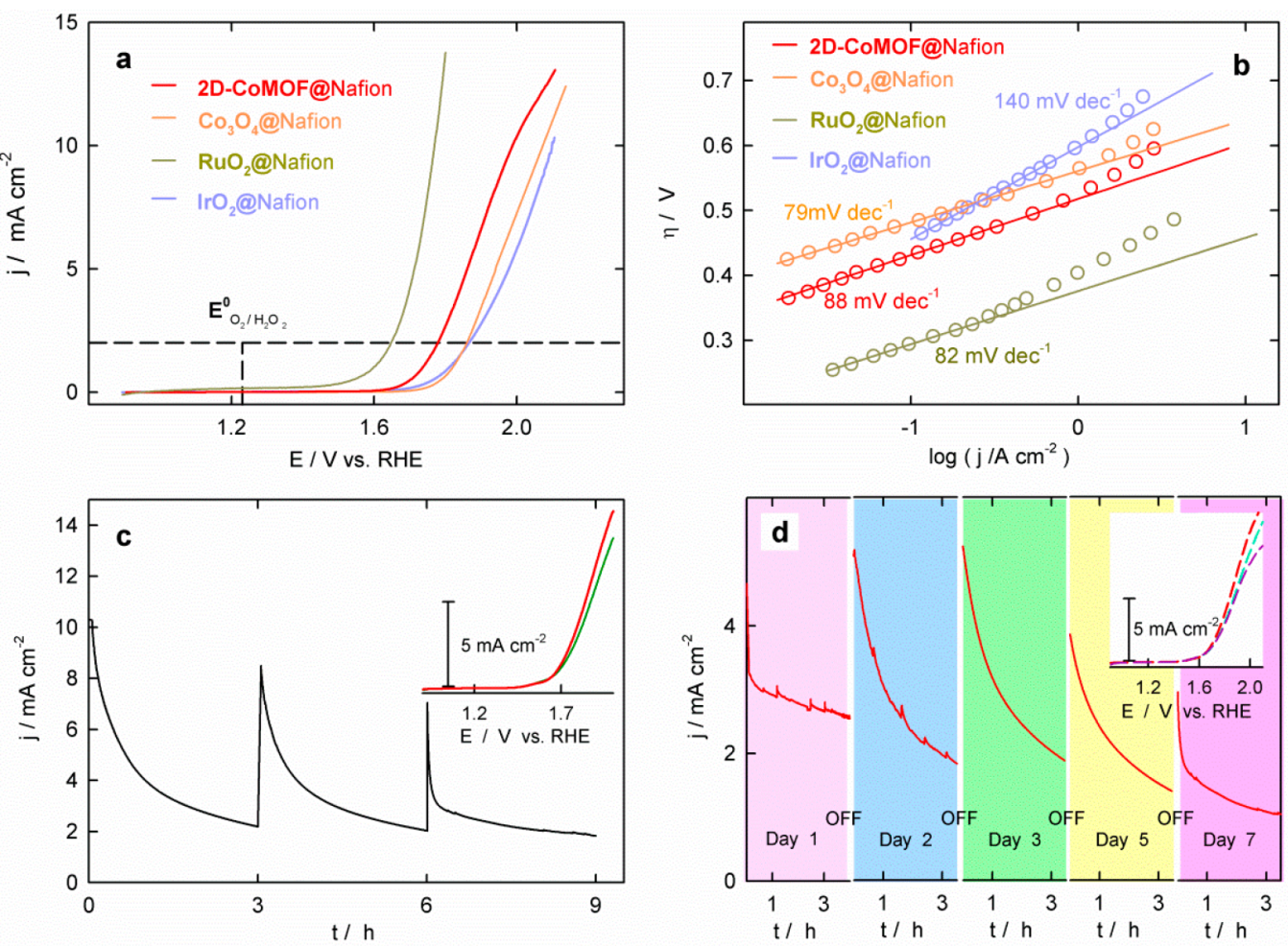

Figure 6. (a) Rotating disk voltammograms recorded at $5 \mathrm{mV} \mathrm{s}^{-1}$ and $1500 \mathrm{rpm}$ and (b) steady-state Tafel plots of a pyrolytic graphite electrode modified with the indicated electrocatalyst in a $0.1 \mathrm{M} \mathrm{SPB}$ solution $\mathrm{pH} 7$ at $25^{\circ} \mathrm{C}$. (c) Consecutive chronoamperograms at $1.8 \mathrm{~V}$ vs RHE for a $2 \mathrm{D}-\mathrm{Co}-$ MOF@Nafion-modified graphite electrode in an aqueous $0.1 \mathrm{M} \mathrm{SPB}$ solution with $\mathrm{pH} 7$ at $25^{\circ} \mathrm{C}$. Inset plot: rotating disk voltammograms measured at $5 \mathrm{mV} \mathrm{s}^{-1}$ before (red line) and after (green line) the chronoamperometric experiment. (d) Chronoamperograms at 1.8 V vs RHE for a 2D-Co-MOF@ Nafion-modified graphite electrode in an aqueous $0.1 \mathrm{M} \mathrm{SPB}$ solution with $\mathrm{pH} 7$ at $25^{\circ} \mathrm{C}$ for several on/off reaction cycles. Inset plot: rotating disk voltammograms measured at $5 \mathrm{mV} \mathrm{s}^{-1}$ after catalytic run at day 1 (red line), day 3 (green line), and day 7 (purple line).

neutral solution, steady-state polarization curves were measured by means of rotating disk voltammetry. Figure 6a depicts the polarization curves recorded in neutral media with $2 \mathrm{D}$-Co-MOF and the typical OER electrocatalysts $\mathrm{RuO}_{2}, \mathrm{IrO}_{2}$, and $\mathrm{Co}_{3} \mathrm{O}_{4}$, all of them codeposited with Nafion onto a graphite electrode. The electrocatalytic onset potentials were $1.73,1.65,1.77$, and $1.84 \mathrm{~V}$ vs RHE for 2D-Co-MOF, $\mathrm{RuO}_{2}, \mathrm{IrO}_{2}$, and $\mathrm{Co}_{3} \mathrm{O}_{4}$, respectively. The corresponding overpotential values at $2 \mathrm{~mA} \mathrm{~cm}^{-2}$ were 548, 404, 680, and $595 \mathrm{mV}$ for 2D-Co-MOF, $\mathrm{RuO}_{2}, \mathrm{IrO}_{2}$, and $\mathrm{Co}_{3} \mathrm{O}_{4}$, respectively. Note that the overpotential value of $2 \mathrm{D}-\mathrm{Co}-\mathrm{MOF}$ is similar to the values reported for the most active cobalt-MOF based catalysts at pH 7 (Table S6).

The kinetics of the electrocatalytic OER mediated by the different electrocatalysts was assessed from the corresponding Tafel plot (Figure $6 \mathrm{~b}$ ) obtained from the chronoamperometric steady-state current density data measured for a sequence of $0.01 \mathrm{~V}$ potential steps. 2D-Co-MOF exhibits a Tafel slope (TS) value of $88 \pm 4 \mathrm{mV} \mathrm{dec}^{-1}$, which is similar to those obtained with $\mathrm{RuO}_{2}$ and $\mathrm{Co}_{3} \mathrm{O}_{4}$ and lower than that of $\mathrm{IrO}_{2}$. To the best of our knowledge, this is the best value among known cobalt MOF catalysts operating in neutral media. The turnover frequency (TOF) value was determined from the expression $\mathrm{TOF}=i / 4 Q$ (where $i$ is the current intensity at a given overpotential and $Q$ is the Faradaic charge under the baseline-corrected voltammetric peak preceding the electrocatalytic branch). A value of $0.034 \mathrm{~s}^{-1}$ was obtained for the TOF of 2D-Co-MOF at an overpotential of $400 \mathrm{mV}$ with $\mathrm{Q}=2.5 \times 10^{-5} \mathrm{C}$ (which corresponds to $2.6 \times$ $10^{-10} \mathrm{~mol}$ of electroactive cobalt). It should be remarked that this TOF value is superior to those reported for similar electrocatalysts $^{27}$ (Table S6). The effect of the amount of electroactive 2D-Co-MOF on the OER electrocatalysis has been also evaluated (Figure S26). An increase in the electroactive cobalt population, obtained by increasing the electrochemical activation time, results in an increase in the electrocatalytic current density at a given potential and a decrease in the corresponding overpotential at a given current density. However, the TOF value determined at an overpotential of $400 \mathrm{mV}$ decreases upon increasing the electroactive population, until it levels off at $\sim 0.035 \mathrm{~s}^{-1}$ for an electroactive population of $2.0 \times 10^{-10} \mathrm{~mol}$. This dependence reveals that not all cobalt centers catalyze the water oxidation reaction at the same level.

The improved activity for the water oxidation reaction of the 2D-Co-MOF@Nafion composite electrocatalyst can be ascribed to both a more efficient charge transport across the catalyst film and a facilitated water nucleophilic attack to the active centers presumably by the presence of nitrogencontaining aromatic equatorial ligands.

The operational stability and long-term durability of the electrocatalyst during the OER have also been explored. First, the chronoamperometric current for the 2D-Co-MOF@Nafionmodified graphite electrode was measured at $1.8 \mathrm{~V}$ (vs RHE) for three consecutive water electrolysis cycles that lasted $9 \mathrm{~h}$ (Figure $6 c)$. The chronoamperograms are characterized by an initial decrease in the current that can be ascribed to the formation of oxygen bubbles at the electrode surface, thereby affecting the water transport inside the multilayered film (Figure S27). Then, the current approaches a steady state value of $\sim 2 \mathrm{~mA} \mathrm{~cm}^{-2}$ in each chronoamperogram, with a negligible decrease in the current density at the end of the $9 \mathrm{~h}$ of operation. The similarity of the polarization curves of fully activated 2D-Co-MOF@ 
Nafion measured before and after the water electrolysis experiment, depicted in the inset plot of Figure $6 c$, reflects the high stability of this material. Moreover, a Faradaic efficiency value of $77 \%$ of $2 \mathrm{D}$-Co-MOF for the OER reaction was determined after extensive water electrolysis, reflecting that the current density mostly originates from water oxidation. Additionally, the analysis of the electrolytic solution after extensive water electrolysis by UPLC-HRMS ( $\mathrm{Q}-\mathrm{ToF})$ reveals the presence of small amounts of pyridine and Nafion (Figure S15), which is consistent with the aforementioned pyridinewater ligand exchange and with a certain composite peeling off during water electrolysis. It should be noted that the solution $\mathrm{pH}$ remains unchanged during water electrolysis.

The recyclability of the catalyst has also been examined along several successive catalytic runs with on/off cycles that lasted 7 days, checking its resistance to activity loss. As can be seen in Figure $6 \mathrm{~d}$, the current density decreases by $50 \%$ after five recycles over 7 days. However, the rotating disk voltammograms recorded after each catalytic run reveal that the 2D-Co-MOF@ Nafion composite retains a remarkable electrocatalytic performance without significant activity loss (inset plot in Figure 6d).

Overall, the long-term stability of delaminated 2D-Co-MOF in the composite is consistent with the absence of significant structural changes in the composite during extensive OER, as described in the previous section.

\section{CONCLUSIONS}

In summary, the synthesis of a new cobalt MOF based on a twolayered core that is strongly connected by intermolecular bonds has been developed. The use of a well-defined cobalt cluster as the starting compound for the synthesis directs the construction of a Co-MOF with an unusual topology. In this MOF, the layered double nanosheets are held together by $\pi-\pi$ stacking interactions between labile pyridine ligands. It has been shown that this material delaminates in the presence of water and that the original 3D layered structure can be regenerated by solvothermal treatment with pyridine, so that the individual nanosheets have associated memory.

Dispersion of the so-synthesized MOF in an alcoholic Nafion solution gives rise to a composite (2D-Co-MOF@Nafion) with good adherence to graphite electrodes and long-term chemical stability. Electrochemical activation of the 2D-Co-MOF@ Nafion-modified electrode improves both ionic migration and electron transfer across the film and promotes the formation of electrocatalytically active cobalt centers. The activated composite exhibits enhanced electrocatalytic activity for water oxidation in neutral media, with a TOF value and robustness superior to those reported for similar electrocatalysts operating under the same experimental conditions. On the basis of the particular topology of the new Co-MOF, with quite distant cobalt centers, and its spectroscopic and electrochemical characterization, a reaction pathway mechanism relying on mononuclear centers is proposed for the cobalt-mediated electrocatalytic OER. Its high electrocatalytic efficiency at neutral $\mathrm{pH}$ is tentatively attributed to the presence of nitrogen-containing aromatic equatorial ligands that presumably facilitate the water nucleophilic attack as in the case of cobalt porphyrins.

\section{ASSOCIATED CONTENT}

\section{(s) Supporting Information}

The Supporting Information is available free of charge at https://pubs.acs.org/doi/10.1021/jacs.0c08882.
Experimental details, synthesis and characterization of 2D-Co-MOF (X-ray diffraction, thermogravimetric analysis, adsorption measurements, and chemical stability), characterization of the electrochemically activated 2DCo-MOF@Nafion composite (X-ray diffraction, Raman spectra, XPS spectra, FESEM images, and EDX analysis), and additional electrochemical characterization of 2DCo-MOF@Nafion composite (voltammetric features and quantification of the electrochemical impedance spectra) (PDF)

Crystallographic data for 2D-Co-MOF (CCDC 1994492) (CIF)

\section{AUTHOR INFORMATION}

\section{Corresponding Author}

Pascual Oña-Burgos - Instituto de Tecnología Química, Universitat Politecnica de VaTencia-Consejo Superior de Investigaciones Cientificas (UPV-CSIC), 46022 Valencia, Spain; Departamento de Quimica y Fisica, Centro de Investigación CIAIMBITAL, Universidad de Almeria, 04120, Spain; ○ orcid.org/0000-0002-2341-7867; Email: pasoabur@ upvnet.upv.es

\section{Authors}

Silvia Gutiérrez-Tarriño - Instituto de Tecnologia Quimica, Universitat Politènica de VaTencia-Consejo Superior de Investigaciones Cientificas (UPV-CSIC), 46022 Valencia, Spain

José Luis Olloqui-Sariego - Departamento de Quimica Fisica, Universidad de Sevilla, 41012 Sevilla, Spain; ํㅏㅇㅣ.org/ 0000-0002-3737-9814

Juan José Calvente - Departamento de Química Física, Universidad de Sevilla, 41012 Sevilla, Spain; 자이.org/ 0000-0002-4229-6204

Guillermo Mínguez Espallargas - Instituto de Ciencia Molecular (ICMol), Universidad de Valencia, 46980 Paterna, Spain; 10 orcid.org/0000-0001-7855-1003

Fernando Rey - Instituto de Tecnología Quimica, Universitat Politécnica de Vatencia-Consejo Superior de Investigaciones Cientificas (UPV-CSIC), 46022 Valencia, Spain; $\odot$ orcid.org/ 0000-0003-3227-5669

Avelino Corma - Instituto de Tecnologia Quimica, Universitat Politécnica de Vatencia-Consejo Superior de Investigaciones Cientificas (UPV-CSIC), 46022 Valencia, Spain; —orcid.org/ 0000-0002-2232-3527

Complete contact information is available at:

https://pubs.acs.org/10.1021/jacs.0c08882

\section{Notes}

The authors declare no competing financial interest.

\section{ACKNOWLEDGMENTS}

Programs Severo Ochoa SEV-2016-0683 and Maria de Maeztu CEX2019-000919-M are gratefully acknowledged. S.G.-T. thanks the MINECO for her FPU Ph.D. contract FPU16/ 02117. P.O.-B. and G.M.E. thank MICCIN for their Ramón y Cajal contracts RYC-2014-16620 and RYC-2013-14386. The authors acknowledge the financial support by the Spanish Government (RTI2018-096399-A-I00, RTI2018-101784-BI00, CTQ2017-89528-P, and CTQ2014-52641-P) and Generalitat Valenciana (PROMETEU/2019/066). In addition, P.O.-B. thanks UPV for his grant PAID-06-18/SP20180172. The 
Electron Microscopy Service of the UPV is acknowledged for their help in sample characterization.

\section{REFERENCES}

(1) Jiao, Y.; Zheng, Y.; Jaroniec, M.; Qiao, S. Z. Design of Electrocatalysts for Oxygen- and Hydrogen-Involving Energy Conversion Reactions. Chem. Soc. Rev. 2015, 44, 2060-2086.

(2) Xia, B. Y.; Yan, Y.; Li, N.; Wu, H. B.; Lou, X. W.; Wang, X. A MetalOrganic Framework-Derived Bifunctional Oxygen Electrocatalyst. Nat. Energy 2016, 1, 1-8.

(3) Ma, T. Y.; Dai, S.; Jaroniec, M.; Qiao, S. Z. Metal-Organic Framework Derived Hybrid $\mathrm{Co}_{3} \mathrm{O}_{4}$-Carbon Porous Nanowire Arrays as Reversible Oxygen Evolution Electrodes. J. Am. Chem. Soc. 2014, 136, 13925-13931.

(4) Piccinin, S.; Sartorel, A.; Aquilanti, G.; Goldoni, A.; Bonchio, M.; Fabris, S. Water Oxidation Surface Mechanisms Replicated by a Totally Inorganic Tetraruthenium-Oxo Molecular Complex. Proc. Natl. Acad. Sci. U. S. A. 2013, 110, 4917-4922.

(5) Yin, Q.; Tan, J. M.; Besson, C.; Geletii, Y. V.; Musaev, D. G.; Kuznetsov, A. E.; Luo, Z.; Hardcastle, K. I.; Hill, C. L. A Fast Soluble Carbon-Free Molecular Water Oxidation Catalyst Based on Abundant Metals. Science 2010, 328, 342-346.

(6) Gao, X.; Zhang, H.; Li, Q.; Yu, X.; Hong, Z.; Zhang, X.; Liang, C.; Lin, Z. Hierarchical $\mathrm{NiCo}_{2} \mathrm{O}_{4}$ Hollow Microcuboids as Bifunctional Electrocatalysts for Overall Water-Splitting. Angew. Chem., Int. Ed. 2016, 55, 6290-6294.

(7) Jin, Y.; Wang, H.; Li, J.; Yue, X.; Han, Y.; Shen, P. K.; Cui, Y. Porous $\mathrm{MoO}_{2}$ Nanosheets as Non-Noble Bifunctional Electrocatalysts for Overall Water Splitting. Adv. Mater. 2016, 28, 3785-3790.

(8) Chen, D.; Chen, C.; Baiyee, Z. M.; Shao, Z.; Ciucci, F. Nonstoichiometric Oxides as Low-Cost and Highly-Efficient Oxygen Reduction/Evolution Catalysts for Low-Temperature Electrochemical Devices. Chem. Rev. 2015, 115, 9869-9921.

(9) Li, Y.; Dai, H. Recent Advances in Zinc-Air Batteries. Chem. Soc. Rev. 2014, 43, 5257-5275.

(10) McCrory, C. C. L.; Jung, S.; Ferrer, I. M.; Chatman, S. M.; Peters, J. C.; Jaramillo, T. F. Benchmarking Hydrogen Evolving Reaction and Oxygen Evolving Reaction Electrocatalysts for Solar Water Splitting Devices. J. Am. Chem. Soc. 2015, 137, 4347-4357.

(11) McCrory, C. C. L.; Jung, S.; Peters, J. C.; Jaramillo, T. F. Benchmarking Heterogeneous Electrocatalysts for the Oxygen Evolution Reaction. J. Am. Chem. Soc. 2013, 135, 16977-16987.

(12) Minguzzi, A.; Fan, F. R. F.; Vertova, A.; Rondinini, S.; Bard, A. J. Dynamic Potential-PH Diagrams Application to Electrocatalysts for Water Oxidation. Chem. Sci. 2012, 3, 217-229.

(13) Duan, J.; Chen, S.; Jaroniec, M.; Qiao, S. Z. Heteroatom-Doped Graphene-Based Materials for Energy-Relevant Electrocatalytic Processes. ACS Catal. 2015, 5, 5207-5234.

(14) Ma, T. Y.; Ran, J.; Dai, S.; Jaroniec, M.; Qiao, S. Z. PhosphorusDoped Graphitic Carbon Nitrides Grown In Situ on Carbon-Fiber Paper: Flexible and Reversible Oxygen Electrodes. Angew. Chem., Int. Ed. 2015, 54, 4646-4650.

(15) Ma, T. Y.; Cao, J. L.; Jaroniec, M.; Qiao, S. Z. Interacting Carbon Nitride and Iitanium Carbide Nanosheets for High-Performance Oxygen Evolution. Angew. Chem., Int. Ed. 2016, 55, 1138-1142.

(16) Davenport, T. C.; Ahn, H. S.; Ziegler, M. S.; Tilley, T. D. A Molecular Structural Analog of Proposed Dinuclear Active Sites in Cobalt-Based Water Oxidation Catalysts. Chem. Commun. 2014, 50, 6326-6329.

(17) Passard, G.; Ullman, A. M.; Brodsky, C. N.; Nocera, D. G. Oxygen Reduction Catalysis at a Dicobalt Center: The Relationship of Faradaic Efficiency to Overpotential. J. Am. Chem. Soc. 2016, 138, $2925-2928$.

(18) Kanan, M. W.; Nocera, D. G. In Situ Formation of an OxygenEvolving Catalyst in Neutral Water Containing Phosphate and $\mathrm{Co}^{2+}$. Science 2008, 321, 1072-1075.

(19) Ling, T.; Yan, D. Y.; Jiao, Y.; Wang, H.; Zheng, Y.; Zheng, X.; Mao, J.; Du, X. W.; Hu, Z.; Jaroniec, M.; Qiao, S. Z. Engineering Surface
Atomic Structure of Single-Crystal Cobalt (II) Oxide Nanorods for Superior Electrocatalysis. Nat. Commun. 2016, 7, 1-8.

(20) Chen, S.; Qiao, S. Z. Hierarchically Porous Nitrogen-Doped Graphene_ $\mathrm{NiCo}_{2} \mathrm{O}_{4}$ Hybrid Paper as an Advanced Electrocatalytic Water-Splitting Material. ACS Nano 2013, 7, 10190-10196.

(21) Zhu, Y. P.; Ma, T. Y.; Jaroniec, M.; Qiao, S. Z. Self-Templating Synthesis of Hollow $\mathrm{Co}_{3} \mathrm{O}_{4}$ Microtube Arrays for Highly Efficient Water Electrolysis. Angew. Chem., Int. Ed. 2017, 56, 1324-1328.

(22) Meng, C.; Ling, T.; Ma, T. Y.; Wang, H.; Hu, Z.; Zhou, Y.; Mao, J.; Du, X. W.; Jaroniec, M.; Qiao, S. Z. Atomically and Electronically Coupled Pt and $\mathrm{CoO}$ Hybrid Nanocatalysts for Enhanced Electrocatalytic Performance. Adv. Mater. 2017, 29, 1604607-1606613.

(23) Hu, H.; Guan, B.; Xia, B.; Lou, X. W. Designed Formation of $\mathrm{Co}_{3} \mathrm{O}_{4} / \mathrm{NiCo}_{2} \mathrm{O}_{4}$ Double-Shelled Nanocages with Enhanced Pseudocapacitive and Electrocatalytic Properties. J. Am. Chem. Soc. 2015, 137, $5590-5595$.

(24) Jin, H.; Wang, J.; Su, D.; Wei, Z.; Pang, Z.; Wang, Y. In Situ Cobalt-Cobalt Oxide/N-Doped Carbon Hybrids As Superior Bifunctional Electrocatalysts for Hydrogen and Oxygen Evolution. J. Am. Chem. Soc. 2015, 137, 2688-2694.

(25) Zhang, Y.; Ouyang, B.; Xu, J.; Jia, G.; Chen, S.; Rawat, R. S.; Fan, H. J. Rapid Synthesis of Cobalt Nitride Nanowires: Highly Efficient and Low-Cost Catalysts for Oxygen Evolution. Angew. Chem., Int. Ed. 2016, $55,8670-8674$

(26) Wang, W.; Xu, X.; Zhou, W.; Shao, Z. Recent Progress in Metal Organic Frameworks for Applications in Electrocatalytic and Photocatalytic Water Splitting. Adv. Sci. 2017, 4, 1600371.

(27) Liao, P. Q.; Shen, J. Q.; Zhang, J. P. Metal-Organic Frameworks for Electrocatalysis. Coord. Chem. Rev. 2018, 373, 22-48.

(28) Gong, Y.; Hao, Z.; Meng, J.; Shi, H.; Jiang, P.; Zhang, M.; Lin, J. Two Co ${ }^{\text {II }}$ Metal-Organic Frameworks Based on a Multicarboxylate Ligand as Electrocatalysts for Water Splitting. ChemPlusChem 2014, 79, 266-277.

(29) Gong, Y.; Shi, H. F.; Jiang, P. G.; Hua, W.; Lin, J. H. Metal(II)Induced Coordination Polymer Based on 4-(5-(Pyridin-4-yl)-4H1,2,4-triazol-3-yl)benzoate as an Electrocatalyst for Water Splitting. Cryst. Growth Des. 2014, 14, 649-657.

(30) Wang, H.; Yin, F.; Li, G.; Chen, B.; Wang, Z. Preparation, characterization and bifunctional catalytic properties of $\mathrm{MOF}(\mathrm{Fe} / \mathrm{Co})$ catalyst for oxygen reduction/evolution reactions in alkaline electrolyte. Int. J. Hydrogen Energy 2014, 39, 16179-16186.

(31) Xu, Q.; Li, H.; Yue, F.; Chi, L.; Wang, J. Nanoscale Cobalt Metal-Organic Framework as a Catalyst for Visible Light-Driven and Electrocatalytic Water Oxidation. New J. Chem. 2016, 40, 3032-3035.

(32) Lu, X. F.; Liao, P. Q.; Wang, J. W.; Wu, J. X.; Chen, X. W.; He, C. T.; Zhang, J. P.; Li, G. R.; Chen, X. M. An Alkaline-Stable, Metal Hydroxide Mimicking Metal-Organic Framework for Efficient Electrocatalytic Oxygen Evolution. J. Am. Chem. Soc. 2016, 138, 8336-8339.

(33) Zhao, S.; Wang, Y.; Dong, J.; He, C. T.; Yin, H.; An, P.; Zhao, K.; Zhang, X.; Gao, C.; Zhang, L.; Lv, J.; Wang, J.; Zhang, J.; Khattak, A. M.; Khan, N. A.; Wei, Z.; Zhang, J.; Liu, S.; Zhao, H.; Tang, Z. Ultrathin metal-organic framework nanosheets for electrocatalytic oxygen evolution. Nat. Energy 2016, 1, 16184.

(34) Shen, J. Q.; Liao, P. Q.; Zhou, D. D.; He, C. T.; Wu, J. X.; Zhang W. X.; Zhang, J. P.; Chen, X. M. Modular and Stepwise Synthesis of a Hybrid Metal-Organic Framework for Efficient Electrocatalytic Oxygen Evolution. J. Am. Chem. Soc. 2017, 139, 1778-1781.

(35) Song, J.; Zhu, G.; Xu, B. Z.; Fu, S.; Engelhard, M. H.; Ye, R.; Du, D.; Beckman, S. P.; Li, Y. Bimetallic Cobalt-Based Phosphide Zeolitic Imidazolate Framework: CoPx Phase-Dependent Electrical Conductivity and Hydrogen Atom Adsorption Energy for Efficient Overall Water Splitting. Adv. Energy Mater. 2017, 7, 1601555.

(36) Jiang, Z.; Ge, L.; Zhuang, L.; Li, M.; Wang, Z.; Zhu, Z. FineTuning the Coordinatively Unsaturated Metal Sites of Metal- Organic Frameworks by Plasma Engraving for Enhanced Electrocatalytic Activity. ACS Appl. Mater. Interfaces 2019, 11, 44300-44307.

(37) Xue, Z.; Liu, K.; Liu, Q.; Li, Y.; Li, M.; Su, C.-Y.; Ogiwara, N.; Kobayashi, H.; Kitagawa, H.; Liu, M.; Li, G. Missing-linker metal- 
organic frameworks for oxygen evolution reaction. Nat. Commun. 2019, $10,5048$.

(38) Gutiérrez Tarriño, S.; Olloqui-Sariego, J. L.; Calvente, J. J.; Palomino, M.; Minguez Espallargas, G.; Jorda, J. L.; Rey, F.; OñaBurgos, P. Cobalt Metal-Organic Framework Based on Two Dinuclear Secondary Building Units for Electrocatalytic Oxygen Evolution. ACS Appl. Mater. Interfaces 2019, 11, 46658.

(39) Zou, Z.; Wang, T.; Zhao, X.; Jiang, W. J.; Pan, H.; Gao, D.; Xu, C. Expediting in-Situ Electrochemical Activation of Two-Dimensional Metal-Organic Frameworks for Enhanced OER Intrinsic Activity by Iron Incorporation. ACS Catal. 2019, 9, 7356-7364.

(40) Gao, Z.; Yu, Z. W.; Liu, F. Q.; Yang, C.; Yuan, Y. H.; Yu, Y.; Luo, F. Stable Iron Hydroxide Nanosheets@Cobalt-Metal-OrganicFramework Heterostructure for Efficient Electrocatalytic Oxygen Evolution. ChemSusChem 2019, 12, 4623-4628.

(41) Li, W.; Watzele, S.; El-Sayed, H. A.; Liang, Y.; Kieslich, G.; Bandarenka, A. S.; Rodewald, K.; Rieger, B.; Fischer, R. A. Unprecedented High Oxygen Evolution Activity of Electrocatalysts Derived from Surface-Mounted Metal-Organic Frameworks. J. Am. Chem. Soc. 2019, 141, 5926-5933.

(42) Takaishi, S.; Hosoda, M.; Kajiwara, T.; Miyasaka, H.; Yamashita, M.; Nakanishi, Y.; Kitagawa, Y.; Yamaguchi, K.; Kobayashi, A.; Kitagawa, H. Electroconductive Porous Coordination Polymer $\mathrm{Cu}$ $\left[\mathrm{Cu}(\mathrm{Pdt})_{2}\right]$ Composed of Donor and Acceptor Building Units. Inorg. Chem. 2009, 48, 9048-9050.

(43) Miyasaka, H. Control of Charge Transfer in Donor/Acceptor Metal-Organic Frameworks. Acc. Chem. Res. 2013, 46, 248-257.

(44) Huo, P.; Chen, T.; Hou, J. Le; Yu, L.; Zhu, Q. Y.; Dai, J. Ligandto-Ligand Charge Transfer within Metal-Organic Frameworks Based on Manganese Coordination Polymers with TetrathiafulvaleneBicarboxylate and Bipyridine Ligands. Inorg. Chem. 2016, 55, 64966503.

(45) Leong, C. F.; Chan, B.; Faust, T. B.; D’Alessandro, D. M. Controlling Charge Separation in a Novel Donor-Acceptor MetalOrganic Framework via Redox Modulation. Chem. Sci. 2014, 5, 47244728.

(46) Darago, L. E.; Aubrey, M. L.; Yu, C. J.; Gonzalez, M. I.; Long, J. R. Electronic Conductivity, Ferrimagnetic Ordering, and Reductive Insertion Mediated by Organic Mixed-Valence in a Ferric Semiquinoid Metal-Organic Framework. J. Am. Chem. Soc. 2015, 137, 15703-15711.

(47) Murase, R.; Leong, C. F.; D’Alessandro, D. M. Mixed Valency as a Strategy for Achieving Charge Delocalization in Semiconducting and Conducting Framework Materials. Inorg. Chem. 2017, 56, 1437314382.

(48) Lin, S.; Usov, P. M.; Morris, A. J. The Role of Redox Hopping in Metal-Organic Framework Electrocatalysis. Chem. Commun. 2018, 54, 6965-6974.

(49) Dissegna, S.; Epp, K.; Heinz, W. R.; Kieslich, G.; Fischer, R. A. Defective Metal-Organic Frameworks. Adv. Mater. 2018, 30, 1704501.

(50) Shearer, G. C.; Chavan, S.; Bordiga, S.; Svelle, S.; Olsbye, U.; Lillerud, K. P. Defect engineering: tuning the porosity and composition of the metal-organic framework UiO-66 via modulated synthesis. Chem. Mater. 2016, 28, 3749-3761.

(51) Tao, L.; Lin, C.-Y.; Dou, S.; Feng, S.; Chen, D.; Liu, D.; Huo, J.; Xia, Z.; Wang, S. Creating Coordinatively Unsaturated Metal Sites in Metal-Organic-Frameworks as Efficient Electrocatalysts for the Oxygen Evolution Reaction: Insights into the Active Centers. Nano Energy 2017, 41, 417-425.

(52) Slater, B.; Wang, Z. R.; Jiang, S. X.; Hill, M. R.; Ladewig, B. P. Missing linker defects in a homochiral metal-organic framework: tuning the chiral separation capacity. J. Am. Chem. Soc. 2017, 139, 1832218327.

(53) Yuan, S.; Qin, J. S.; Zou, L.; Chen, Y. P.; Wang, X.; Zhang, Q.; Zhou, H. C. Thermodynamically guided synthesis of mixed-linker ZrMOFs with enhanced tunability. J. Am. Chem. Soc. 2016, 138, 66366642.

(54) Fang, Z.; Dürholt, J. P.; Kauer, M.; Zhang, W.; Lochenie, C.; Jee, B.; Albada, B.; Metzler-Nolte, N.; Pöppl, A.; Weber, B.; Muhler, M.; Wang, Y.; Schmid, R.; Fischer, R. A. Structural complexity in metal- organic frameworks: simultaneous modification of open metal sites and hierarchical porosity by systematic doping with defective linkers. J. Am. Chem. Soc. 2014, 136, 9627-9636.

(55) Wang, L.-P.; Van Voorhis, T. Direct-Coupling $\mathrm{O}_{2}$ Bond Forming a Pathway in Cobalt Oxide Water Oxidation Catalysts. J. Phys. Chem. Lett. 2011, 2, 2200-2204.

(56) Ullman, A. M.; Brodsky, C. N.; Li, N.; Zheng, S.-L.; Nocera, D. G. Probing Edge Site Reactivity of Oxidic Cobalt Water Oxidation Catalysts. J. Am. Chem. Soc. 2016, 138, 4229-4236.

(57) Mattioli, G.; Giannozzi, P.; Amore Bonapasta, A.; Guidoni, L. Reaction Pathways for Oxygen Evolution Promoted by Cobalt Catalyst. J. Am. Chem. Soc. 2013, 135, 15353-15363.

(58) Smith, P. F.; Hunt, L.; Laursen, A. B.; Sagar, V.; Kaushik, S.; Calvinho, K. U. D.; Marotta, G.; Mosconi, E.; De Angelis, F.; Dismukes, G. C. Water Oxidation by the $\left[\mathrm{Co}_{4} \mathrm{O}_{4}(\mathrm{OAc})_{4}(\mathrm{Py})^{4}\right]^{+}$Cubium Is Initiated by $\mathrm{OH}$ - Addition. J. Am. Chem. Soc. 2015, 137, 15460-15468.

(59) Li, X.; Siegbahn, P. E. M. Water Oxidation Mechanism for Synthetic Co-Oxides with Small Nuclearity. J. Am. Chem. Soc. 2013, 135, 13804-13813.

(60) Pham, H. H.; Cheng, M.-J.; Frei, H.; Wang, L.-W. Surface Proton Hopping and Fast-Kinetics Pathway of Water Oxidation on $\mathrm{Co}_{3} \mathrm{O}_{4}$ (001) Surface. ACS Catal. 2016, 6, 5610-5617.

(61) Soriano-Lopez, J.; Musaev, D. G.; Hill, C. L.; Galan-Mascaros, J. R.; Carbo, J. J.; Poblet, J. M. Tetracobalt-Polyoxometalate Catalysts for Water Oxidation: Key Mechanistic Details. J. Catal. 2017, 350, 56-63.

(62) Wang, D.; Groves, J. T. Efficient Water Oxidation Catalyzed by Homogeneous Cationic Cobalt Porphyrins with Critical Roles for the Buffer Base. Proc. Natl. Acad. Sci. U. S. A. 2013, 110, 15579-15584.

(63) Jayaramulu, K.; Masa, J.; Morales, D. M.; Tomanec, O.; Ranc, V.; Petr, M.; Wilde, P.; Chen, Y. T.; Zboril, R.; Schuhmann, W.; Fischer, R. A. Ultrathin 2D Cobalt Zeolite-Imidazole Framework Nanosheets for Electrocatalytic Oxygen Evolution. Adv. Sci. 2018, 5, 1801029.

(64) Nguyen, A. I.; Ziegler, M. S.; Oña-Burgos, P.; SturzbecherHohne, M.; Kim, W.; Bellone, D. E.; Tilley, T. D. Mechanistic Investigations of Water Oxidation by a Molecular Cobalt Oxide Analogue: Evidence for a Highly Oxidized Intermediate and Exclusive Terminal Oxo Participation. J. Am. Chem. Soc. 2015, 137, 1286512872.

(65) Biesinger, M. C.; Payne, B. P.; Grosvenor, A. P.; Lau, L. W. M.; Gerson, A. R.; Smart, R. S. C. Resolving Surface Chemical States in XPS Analysis of First Row Transition Metals, Oxides and Hydroxides: Cr, $\mathrm{Mn}, \mathrm{Fe}, \mathrm{Co}$ and Ni. Appl. Surf. Sci. 2011, 257, 2717-2730.

(66) Ivanova, T.; Naumkin, A.; Sidorov, A.; Eremenko, I.; Kiskin, M. X-Ray Photoelectron Spectra and Electron Structure of Polynuclear Cobalt Complexes. J. Electron Spectrosc. Relat. Phenom. 2007, 156, 200203.

(67) Okamoto, Y.; Nakano, H.; Imanaka, T.; Teranishi, S. X-Ray Photoelectron Spectroscopic Studies of Catalysts - Supported Cobalt Catalysts. Bull. Chem. Soc. Jpn. 1975, 48, 1163-1168.

(68) Briggs, D.; Gibson, V. A. Direct Observation Of Multiplet Splitting In $2 p$ Photoelectron Peaks Of Cobalt Complexes. Chem. Phys. Lett. 1974, 25, 493-496.

(69) Carver, J. C.; Schweitzer, G. K.; Carlson, T. A. Use of X-Ray Photoelectron Spectroscopy to Study Bonding in Cr, Mn, Fe, and Co Compounds. J. Chem. Phys. 1972, 57, 973-982.

(70) Frost, D. C.; McDowell, C. A.; Woolsey, I. S. X-Ray Photoelectron Spectra of Cobalt Compounds. Mol. Phys. 1974, 27, $1473-1489$.

(71) Fang, H.; Huang, T.; Liang, D.; Qiu, M.; Sun, Y.; Yao, S.; Yu, J.; Dinesh, M. M.; Guo, Z.; Xia, Y.; Mao, S. Prussian blue analog-derived $2 \mathrm{D}$ ultrathin $\mathrm{CoFe}_{2} \mathrm{O}_{4}$ nanosheets as high-activity electrocatalysts for the oxygen evolution reaction in alkaline and neutral media. J. Mater. Chem. A 2019, 7, 7328-7332.

(72) Palomares, A. E.; Patro, J. G.; Rey, F.; Corma. Using the "Memory Effect" of Hydrotalcites for Improving the Catalytic Reduction of Nitrates in Water. J. Catal. 2004, 221, 62-66.

(73) Li, G.; Yang, D.; Chuang, P. A. Defining Nafion Ionomer Roles for Enhancing Alkaline Oxygen Evolution Electrocatalysis. ACS Catal. 2018, 8, 11688-11698. 
(74) Surendranath, Y.; Kanan, M. W.; Nocera, D. G. Mechanistic Studies of the Oxygen Evolution Reaction by a Cobalt-Phosphate Catalyst at Neutral pH. J. Am. Chem. Soc. 2010, 132, 16501-16509.

(75) Costentin, C.; Savéant, J.-M. Cyclic Voltammetry Analysis of Electrocatalytic Films. J. Phys. Chem. C 2015, 119, 12174-12182.

(76) Costentin, C.; Savéant, J.-M. Cyclic Voltammetry of Fast Conducting Electrocatalytic Films. Phys. Chem. Chem. Phys. 2015, 17, 19350-19359.

(77) Inzelt, G.; Lang, G. Model Dependence and Reliability of the Electrochemical Quantities Derived from the Measured Impedance Spectra of Polymer Modified Electrodes. J. Electroanal. Chem. 1994, 378, 39-49.

(78) Bisquert, J.; Garcia-Belmonte, G.; Bueno, P.; Longo, E.; Bulhoes, L. O. S. Impedance of Constant Phase Element (CPE)-Blocked Diffusion in Film Electrodes. J. Electroanal. Chem. 1998, 452, 229-234.

(79) Bisquert, J.; Garcia-Belmonte, G.; Fabregat-Santiago, F.; Bueno, P. R. Theoretical Models for a.c. Impedance of Finite Diffusion Layers Exhibiting Low Frequency Dispersion. J. Electroanal. Chem. 1999, 475, $152-163$.

(80) Huang, J. Diffusion Impedance of Electroactive Materials, Electrolytic Solutions and Porous Electrodes: Warburg Impedance and Beyond. Electrochim. Acta 2018, 281, 170-188.

(81) Lasia, A. In Electrochemical Impedance Spectroscopy and Its Applications; Conway, B. E., Bockris, J., White, R. E., Eds.; Kluwer Academic/Plenum Publishers: New York, 1999, Modern Aspects of Electrochemistry Vol. 32, p 143.

(82) Kenley, R. A.; Fleming, R. H.; Laine, R. M.; Tse, D. S.; Winterle, J. S. Cobalt(III) Complex Catalyzed Hydrolysis of Phosphorus Esters. Inorg. Chem. 1984, 23, 1870-1876.

(83) Lei, H.; Han, A.; Li, F.; Zhang, M.; Han, Y.; Du, P.; Lai, W.; Cao, R. Electrochemical, Spectroscopic and Theoretical Studies of a Simple Bifunctional Cobalt Corrole Catalyst for Oxygen Evolution and Hydrogen Production. Phys. Chem. Chem. Phys. 2014, 16, 1883-1893. (84) Lieb, D.; Zahl, A.; Wilson, E. F.; Streb, C.; Nye, L. C.; Meyer, K.; Ivanovic-Burmazovic, I. Water Exchange Reactivity and Stability of Cobalt Polyoxometalates under Catalytically Relevant $\mathrm{pH}$ Conditions: Insight into Water Oxidation Catalysis. Inorg. Chem. 2011, 50, 90539058.

(85) Nazeeruddin, M. K.; Zakeeruddin, S. M.; Humphry-Baker, R.; Jirousek, M.; Liska, P.; Vlachopoulos, N.; Shklover, V.; Fischer, C. H.; Gratzel, M. Acid-Base Equilibria of (2,2'-Bipyridyl-4,4'-dicarboxylic acid)ruthenium(II) Complexes and the Effect of Protonation on Charge-Transfer Sensitization of Nanocrystalline Titania. Inorg. Chem. 1999, 38, 6298-6305.

(86) Lõkov, M.; Tshepelevitsh, S.; Heering, A.; Plieger, P. G.; Vianello, R; Leito, I. On the Basicity of Conjugated Nitrogen Heterocycles in Different Media. Eur. J. Org. Chem. 2017, 2017, $4475-4489$.

(87) Nazeeruddin, M. K.; Kalyanasundaram, K. Acid-Base Behavior in the Ground and Excited States of Ruthenium(II) Complexes Containing Tetraimines or Dicarboxybipyridines as Protonatable Ligands. Inorg. Chem. 1989, 28, 4251-4259. 